\title{
MicroRNA-98/PTEN/AKT pathway inhibits cell proliferation and malignant progression of hypopharyngeal carcinoma by MTDH
}

\author{
QIWEI WANG ${ }^{1}$, LIJUN TAN ${ }^{2}$, JIANGTAO LIU ${ }^{1}$, JIANNAN ZHAO ${ }^{1}$, XIAOJIE ZHOU ${ }^{1}$ and TIANJIAO YU ${ }^{1}$ \\ Departments of ${ }^{1}$ Otorhinolaryngology and ${ }^{2}$ Oncology, The First Affiliated Hospital of Harbin Medical University, \\ Harbin, Heilongjiang 150001, P.R. China
}

Received June 25, 2018; Accepted November 23, 2018

DOI: $10.3892 /$ or.2018.6904

\begin{abstract}
Laryngeal carcinoma is one of the most common tumors concerning otorhinolaryngology head and neck surgery, however, the pathogenesis of laryngeal carcinoma remains unclear. MicroRNAs (miRNAs) have been reported to play vital roles in the pathogenesis of laryngeal carcinoma Herein, the present study was designed to explore the function and mechanism of miRNA-98 in hypopharyngeal carcinoma. In brief, qRT-PCR, MTT assay, western blot analysis, Transwell assay and luciferase reporter assay were performed. Based on the results, miRNA-98 expression was downregulated in patients with hypopharyngeal carcinoma. Downregulation of miRNA-98 promoted cell growth and migration, and decreased the apoptotic rate of hypopharyngeal carcinoma cells. Overexpression of miRNA-98 increased the apoptotic rate, and inhibited cell growth and migration of hypopharyngeal carcinoma cells. Moreover, luciferase reporter assays revealed that MTDH is a direct target of miRNA-98 and overexpression of miRNA-98 induced the protein expression of PTEN and suppressed that of PI3K and p-Akt. si-MTDH attenuated the anticancer effects of miRNA-98 on hypopharyngeal carcinoma via the PTEN/AKT pathway. To the best of our knowledge, the present study confirmed for the first time that miRNA-98 inhibits hypopharyngeal carcinoma cell proliferation and induces apoptosis via the PTEN/AKT pathway by MTDH.
\end{abstract}

\section{Introduction}

Head and neck tumors are major cancers threatening human life, whose morbidity ranks sixth among all cancer types (1). Laryngeal carcinoma is also one of the most common head and neck tumors, with the morbidity ranking second among

Correspondence to: Professor Jiangtao Liu, Department of Otorhinolaryngology, The First Affiliated Hospital of Harbin Medical University, 23 Youzheng Street, Nangang, Harbin, Heilongjiang 150001, P.R. China

E-mail: 1jt89103@aliyun.com

Key words: hypopharyngeal carcinoma, microRNA-98, PTEN, AKT, MTDH all respiratory tract tumors (1). The most common subtype of laryngeal carcinoma is squamous cell carcinoma, which accounts for about $95 \%$ of all cases. Industrial development and increased air pollution have resulted in the increasing morbidity of laryngeal carcinoma (2). Research suggests an annual rate of increase of $25 \%$ of the incidence of this tumor (2). Laryngeal carcinoma is commonly diagnosed in middle-aged and elderly males. The global morbidity of laryngeal carcinoma in males was estimated to be 5.1/100,000 in 2008, and the mortality in males is about 2.2/100,000 (3). New surgical methods, chemotherapeutics and more advanced radiotherapy strategies have been applied for the treatment of laryngeal carcinoma in the recent 30 years (3). However, according to the latest research, the overall survival of laryngeal carcinoma patients has not improved (4). Unfortunately, the overall survival has shown a decreasing trend, which reaches only approximately $50 \%$. Moreover, the survival rate for advanced laryngeal carcinoma is even as low as $30-40 \%$ (4).

Laryngeal carcinoma is one of the common tumors concerning otorhinolaryngology head and neck surgery. Its genesis and development are associated with aberrantly expressed microRNAs (miRs or miRNAs) (5). Existing research results have indicated the aberrant expression of multiple miRNAs in laryngeal carcinoma tissues. Moreover, the number of downregulated miRNAs is greater than that of upregulated ones (5). miRNA-related research suggests that, miRNAs will serve as novel molecular markers for diagnosing laryngeal carcinoma, similar to other molecular markers for laryngeal carcinoma (6). They may aid in the early diagnosis, prediction of prognosis and recurrence and follow-up of laryngeal carcinoma. In addition, miRNAs are potential targets for laryngeal carcinoma treatment (5). Notably, they present new application prospects for designing drugs for the targeted treatment of laryngeal carcinoma. Therefore, they will bring hope to laryngeal carcinoma patients (5).

The PI3K/Akt pathway is an important growth factor pathway in vivo. It can activate the anti-cell apoptosis mechanism, glucose metabolism and protein synthesis (7), thus, promoting cell growth and proliferation (8). Abnormality of such a signal transduction pathway can lead to abnormal cell proliferation and induce tumor growth. This is common in malignant tumor tissues, such as human ovarian, breast, pancreatic, lung and colon cancer. The PI3K/Akt pathway is verified to be involved in the genesis and development of most 
tumors (9). Therefore, the PI3K/Akt pathway is considered to be the primary pathway for cancer cell survival. It is also named the anti-apoptosis pathway (9).

MTDH is also called stellate cell upregulated gene 1, 3D3 and lysine-rich CEACAM 1-related protein (10). It is a newly discovered oncogene. Research indicates that MTDH is markedly upregulated in numerous tumor cells and can activate multiple signaling pathways. These cancer types include breast, prostate, esophageal, lung cancer, melanoma and glioblastoma (10). Thus, it participates in tumor growth and proliferation, angiogenesis, invasion and metastasis and drug resistance. Meanwhile, it is closely related to tumor progression (10).

PTEN is a tumor-suppressor gene with phosphatase activity. The protein encoded by PTEN possesses the features and functions of phosphoesterase (11). Meanwhile, it shows extensive homology to cytoskeletal protein, and is highly expressed in epithelial cells. PTEN expression product can neutralize the protein tyrosine kinases (PTKs) and can inhibit tumor cell growth. Moreover, it can regulate tumor cell invasion and metastasis through interaction with local adhesion (11). The present study aimed to explore the function and mechanism of miRNA-98 in hypopharyngeal carcinoma.

\section{Materials and methods}

Clinical specimens. A total of 42 patients with hypopharyngeal carcinoma (male $=40$ and female $=42$ ) and 12 healthy volunteers (male $=6$ and female $=6$ ) were enrolled at The First Affiliated Hospital of Harbin Medical University for participation in this study from May 2012 to September 2012. The study protocol was approved by the Human Research Ethics Committee of The First Affiliated Hospital of Harbin Medical University, and all patients provided written informed consent prior to participation in the study. Peripheral blood was collected and centrifuged at $2,000 \mathrm{x}$ g for $10 \mathrm{~min}$ at $4^{\circ} \mathrm{C}$. Serum was stored at $-80^{\circ} \mathrm{C}$.

$q R T-P C R$ analysis. Total RNA was extracted from serum or cell samples with TRIzol reagent (Thermo Fisher Scientific, Inc., Waltham, MA, USA). RNA (1 $\mu \mathrm{g})$ was reverse transcribed into cDNA using SuperScript II reverse transcriptase (code DRR037A; Takara Biotechnology Co., Ltd., Dalian, China). qRT-PCR was performed using the 7500 Real-Time PCR system (Applied Biosystems, Mannheim, Germany) at conditions of $95^{\circ} \mathrm{C}$ for $10 \mathrm{~min} ; 40$ cycles of denaturation at $95^{\circ} \mathrm{C}$ for $30 \mathrm{sec}$, annealing at $58^{\circ} \mathrm{C}$ for $30 \mathrm{sec}$ and extension at $72^{\circ} \mathrm{C}$ for $70 \mathrm{sec}$. Primer sequences for RT-qPCR: miRNA-98: F, 5'-GGGGTGAGGTAGTAAGTTGT-3' and R, 5'-TGGGTG TCGTGGAGTC-3'; U6: F, 5'-CTCGCTTCGGCAGCACA-3' and R, 5'-AACGCTTCACGAATTTGCGT-3'. Relative expression of the miRNA was calculated using the comparative $\mathrm{Ct}$ method (12). The high expression of miRNA-98 in patients with hypopharyngeal carcinoma was $\geq 0.4$ of healthy volunteers; low expression of miRNA-98 in patients with hypopharyngeal carcinoma was $<0.4$ of healthy volunteers.

Gene expression profiling. Total RNA was reverse transcribed into cDNA and hybridized to Affymetrix HG-U133 Plus 2.0 GeneChip arrays (Affymetrix GeneChip; Affymetrix; Thermo
Fisher Scientific, Inc.). Data were analyzed through the use of the Database for Annotation, Visualization and Integrated Discovery (DAVID Database; https://david.ncifcrf.gov/) and Qiagen's Ingenuity Pathway Analysis (IPA; Qiagen, Redwood City, CA, USA).

Cell lines and culture conditions. The hypopharyngeal carcinoma cell line FaDu was obtained from the Shanghai Cell Bank of the Chinese Academy of Sciences. The cells were cultured in Dulbecco's modified Eagle's medium (DMEM, Thermo Fisher Scientific, Inc.) containing $10 \%$ fetal calf serum (FCS, Gibco; Thermo Fisher Scientific, Inc.), 100 U/ml penicillin and $100 \mathrm{mg} / \mathrm{ml}$ streptomycin, at $37^{\circ} \mathrm{C}$ in $5 \% \mathrm{CO}_{2}$.

Transfection. MTDH plasmid, microRNA-98, anti-microRNA-98 and negative control mimics were transfected into the cells using Lipofectamine 2000 reagent (Invitrogen; Thermo Fisher Scientific, Inc.) at $37^{\circ} \mathrm{C}$. After transfection for $4 \mathrm{~h}$, old medium was removed and new DMEM was added into the cells. Next, after transfection for $4 \mathrm{~h}$, PTEN inhibitor (20 nM, VO-Ohpic trihydrate) was added into cells for $44 \mathrm{~h}$.

MTT assay. MTT $(5 \mathrm{mg} / \mathrm{ml}, 20 \mu \mathrm{l})$ was added into the cells for $4 \mathrm{~h}$ at $37^{\circ} \mathrm{C}$. Old medium was replaced with dimethyl sulfoxide (DMSO) $(150 \mu \mathrm{l})$ which was added into the cells for $20 \mathrm{~min}$ at $37^{\circ} \mathrm{C}$. The absorbance was measured by spectrophotometry with a microplate reader (model 680; Bio-Rad Laboratories, Hercules, CA, USA) at $490 \mathrm{~nm}$.

LDH assay. Cells were assessed for LDH activity using LDH activity kit (C0016; Beyotime Institute of Biotechnology, Haimen, China). The absorbance was measured by spectrophotometry with a microplate reader (model 680; Bio-Rad Laboratories) at $450 \mathrm{~nm}$.

Transwell assay. The Transwell assay was used to measure the invasion capability of the transfected FaDu cells. FaDu cells $\left(1 \times 10^{5}\right.$ cells/well $)$ were added to the upper chambers of Transwell inserts (BD Biosciences, Franklin Lakes, NJ, USA; $8-\mu$ m pore size). DMEM with $10 \%$ FBS was added to the lower chambers. After $48 \mathrm{~h}$ of incubation, cells were fixed with $100 \%$ methanol for $10 \mathrm{~min}$ and stained with $0.2 \%$ crystal violet for $20 \mathrm{~min}$ at room temperature. Image was captured using a Leica microscope image system at x100 magnification (Leica Microsystems, Mannheim, Germany).

Determination of apoptosis by flow cytometry. Cells were washed with phosphate-buffered saline (PBS) and fixed using 4\% paraformaldehyde for $15 \mathrm{~min}$ and stained with Annexin V/PI assay (cat. no. 88-8007; eBioscience, Inc., San Diego, CA, USA) for $15 \mathrm{~min}$ in darkness. The apoptosis rate was determined using FACSCalibur flow cytometer (BD Biosciences, Sydney, Australia).

Western blot analysis. Cells were collected and lysed in radioimmunoprecipitation assay buffer (RIPA) and the protein samples $(50 \mu \mathrm{g})$ were separated by electrophoresis using 10\% SDS-PAGE and then transferred to a polyvinylidene difluoride (PVDF) membrane. The membrane was blocking with Tris-buffered saline (TBS) containing 5\% non-fat milk and 0.1\% Tween-20 at 

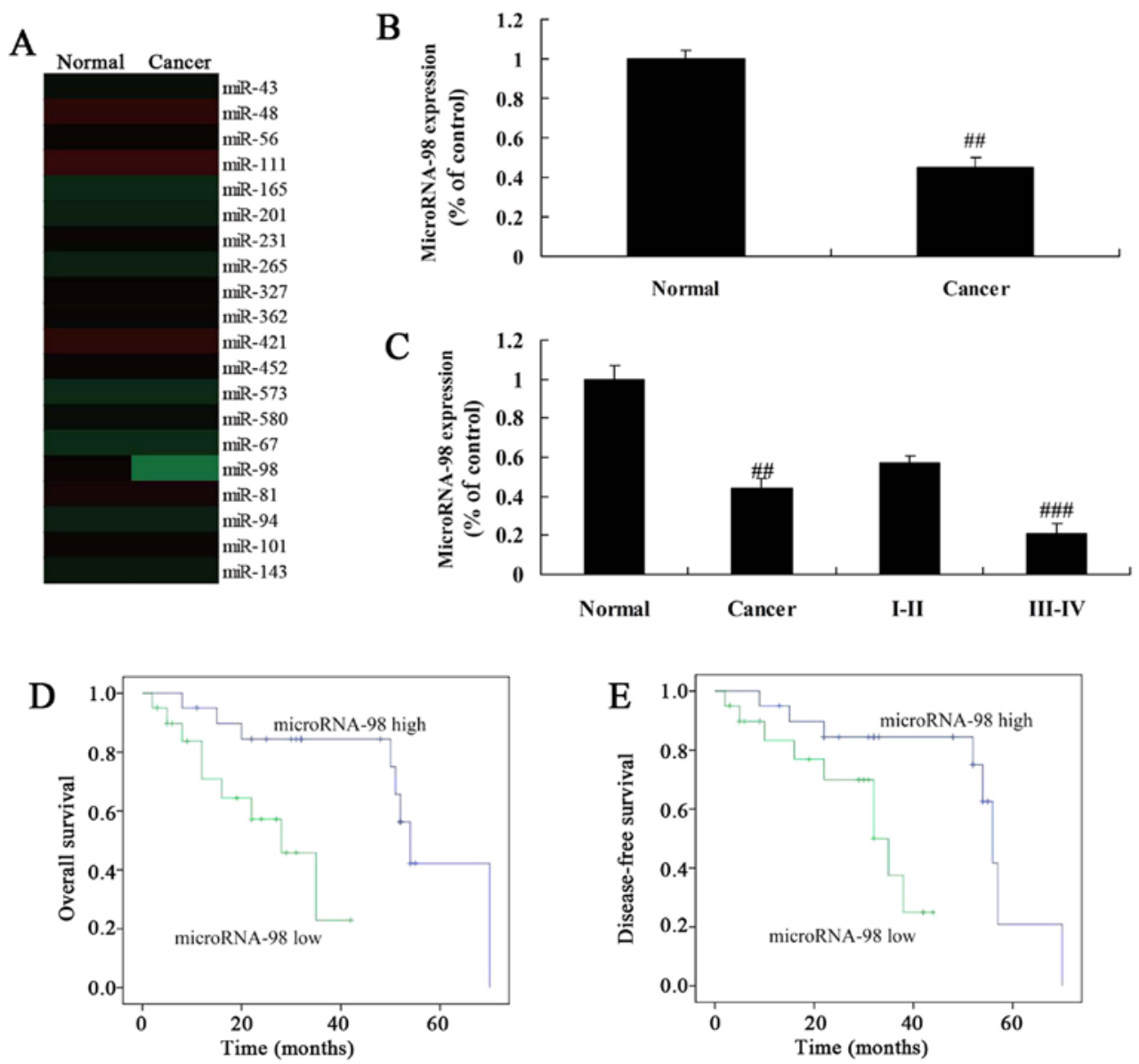

Figure 1. MicroRNA-98 (miR-98) expression in hypopharyngeal carcinoma. (A) Heat map of the gene chip for microRNA expression in hypopharyngeal carcinoma. (B and C) miR-98 expression in hypopharyngeal carcinoma and normal tissues. (D and E) OS and DFS of hypopharyngeal carcinoma patients with low and high expression of miR-98. Normal, normal tissues from 12 healthy volunteers; Cancer, hypopharyngeal carcinoma tissues from 12 patients; I-II, $\mathrm{I}-\mathrm{II}$ stage hypopharyngeal carcinoma tissues; III-IV, III-IV stage hypopharyngeal carcinoma tissues. ${ }^{\# \#} \mathrm{P}<0.01$ compared with the normal tissues; ${ }^{\# \# /} \mathrm{P}<0.01$ compared with the normal tissues.

$37^{\circ} \mathrm{C}$ for $1 \mathrm{~h}$ and subsequently incubated with the primary antibodies at $4^{\circ} \mathrm{C}$ overnight: Bax (dilution 1:1,000; cat. no. sc-6236), PTEN (dilution 1:1,000; cat. no. sc-133197), p-AKT (dilution 1:500; cat. no. sc-7985-R), MTDH (dilution 1:1,000; cat. no. sc-517220) and GAPDH (dilution 1:5,000; cat. no. sc-51631; Santa Cruz Biotechnology, Inc., Dallas, TX, USA). Horseradish peroxidase-linked $\mathrm{IgG}$ was used as the secondary antibody (dilution 1:5,000; cat. no. sc-2004; Santa Cruz Biotechnology) for incubation for $1 \mathrm{~h}$ at $37^{\circ} \mathrm{C}$. Protein blots were visualized by chemiluminescence (NEN Life Science Products, Boston, MA, USA) and analyzed using Image-Pro Plus 6.0 software (Media Cybernetics, Inc., Rockville, MD, USA).

Immunofluorescence staining. FaDu cells were fixed with $4 \%$ paraformaldehyde for $15 \mathrm{~min}$ at room temperature and permeabilized with $0.25 \%$ Triton $\mathrm{X}-100$ for $10 \mathrm{~min}$ at room temperature. Cell was blocked with 5\% BSA in PBS for $1 \mathrm{~h}$ at $37^{\circ} \mathrm{C}$ and incubated overnight at $4{ }^{\circ} \mathrm{C}$ with the antibody for MTDH (dilution 1:100; cat. no. sc-517220; Santa Cruz Biotechnology). Cells were incubated with 555 secondary antibody (dilution 1:1,000; cat. no. sc-362271; Santa Cruz Biotechnology) for $1 \mathrm{~h}$ at $37^{\circ} \mathrm{C}$ and stained with DAPI assay for $15 \mathrm{~min}$ in darkness. Image was captured using a Leica microscope image system (Leica Microsystems).
Luciferase reporter. The 3'-untranslated region (3'-UTR) of MTDH and microRNA-98 mimics were co-transfected using Lipofectamine 2000 reagent (Invitrogen; Thermo Fisher Scientific, Inc.). After 48 h, Renilla luciferase activity was measured using a Dual-Luciferase reporter system (Promega, Madison, WI, USA). Luciferase activity was detected using an Orion II microplate luminometer (Berthold Technologies, Bad Wildbad, Germany).

Statistical analysis. Data are expressed as the mean \pm SEM $(n=3)$. The difference between two independent groups was assessed using the Student's t-test or one-way analysis of variance (ANOVA) and Tukey's post hoc test. Statistical analyses were conducted using SPSS 20.0 software (IBM Corp., Armonk, NY, USA). $\mathrm{P}<0.05$ was considered to indicate a statistically significant result.

\section{Results}

Expression of miRNA-98 in hypopharyngeal carcinoma. Both GeneChip and qPCR were used to detect the expression of microRNAs in the normal and cancer tissues. The expression of miRNA-98 was significantly downregulated in patients with hypopharyngeal carcinoma, compared with that noted in the 
A

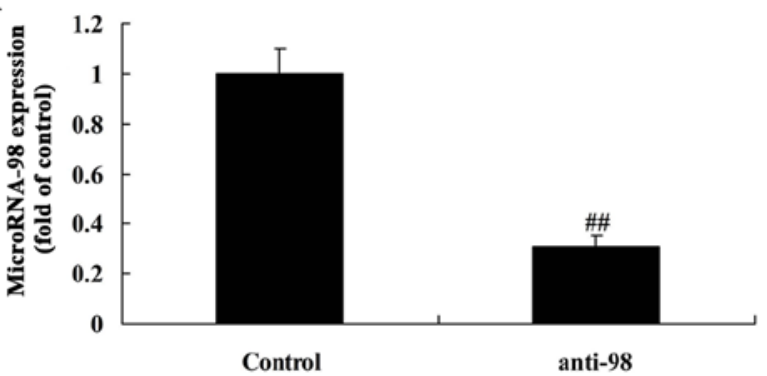

C

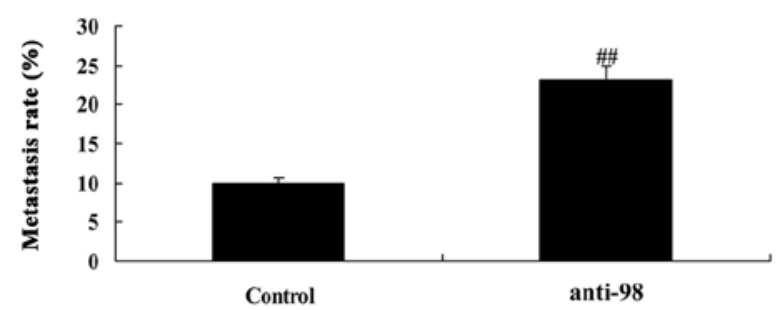

$\mathrm{E}$

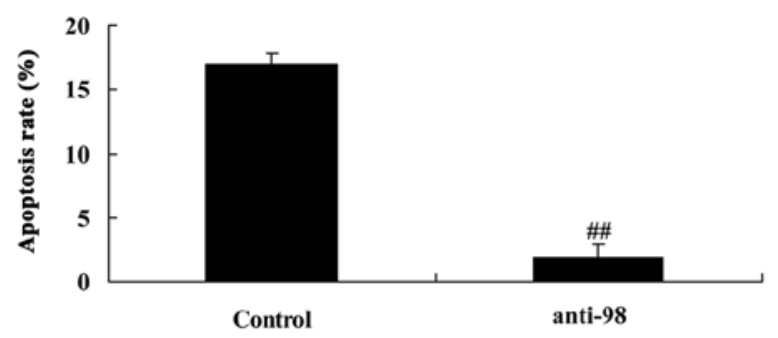

G

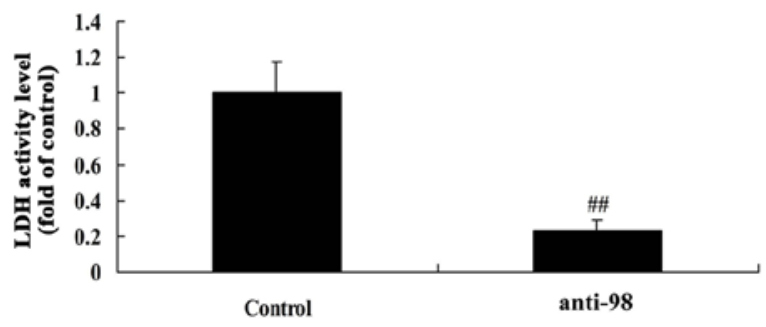

B

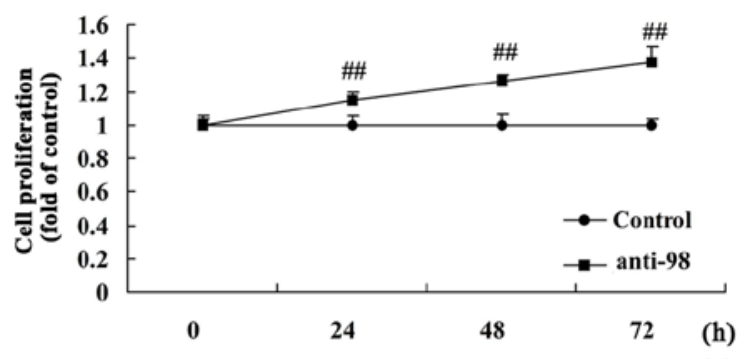

D
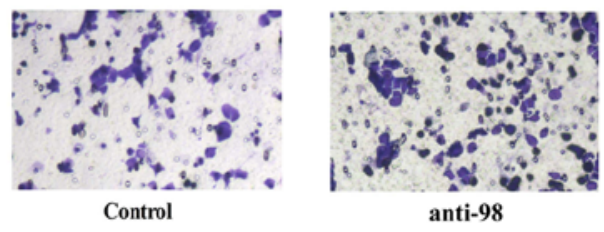

$\mathrm{F}$
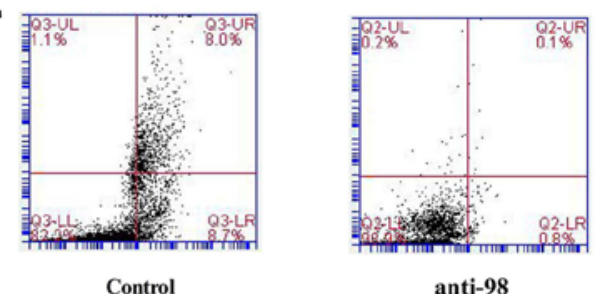

anti-98

$\mathrm{H}$

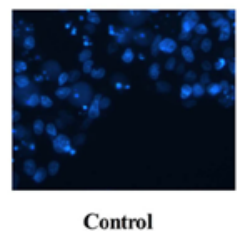

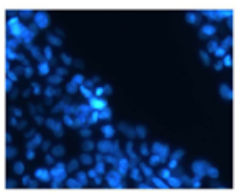

anti-98

Figure 2. Downregulation of microRNA-98 promotes cell growth and migration, and decreased the apoptosis rate of hypopharyngeal carcinoma cells (A) MicroRNA-98 expression, (B) cell proliferation, (C and D) cell migratory, (E and F) the apoptosis rate, (G) LDH activity and (H) DAPI assay. Control, negative control group; anti-98, microRNA-98 downregulated expression group. ${ }^{\# \#} \mathrm{P}<0.01$ compared with the negative control group.

control group (Fig. 1A and B). Meanwhile, the expression of miRNA-98 in patients with stage III-IV hypopharyngeal carcinoma was significantly lower than that in patients with stage I-II hypopharyngeal carcinoma (Fig. 1C). Then, we analyzed the relationship between miRNA-98 expression and the survival rate of patients with hypopharyngeal carcinoma. To this end, Kaplan-Meier survival analysis was carried out to evaluate the relationship of miRNA-98 expression with overall survival (OS) and disease-free survival (DFS) in patients with hypopharyngeal carcinoma. As a result, the OS of patients with hypopharyngeal carcinoma with high expression of miRNA-98 was increased when compared with the OS of patients with hypopharyngeal carcinoma with low expression of miRNA-98 (Fig. 1D). In other words, patients with hypopharyngeal carcinoma with high expression of miRNA-98 harbored obviously prolonged DFS than those with low expression of miRNA-98 (Fig. 1E).
Downregulation of miRNA-98 increases cell growth and migration, and decreases the apoptotic rate of hypopharyngeal carcinoma cells. In order to study the role of miRNA-98 in the proliferation of hypopharyngeal carcinoma cells, FaDu cells were transiently transfected with anti-miRNA-98 mimics. miRNA-98 expression was substantially decreased in the FaDu cells, compared with the negative control-transfected cells (Fig. 2A). Moreover, downregulation of miRNA-98 increased cell viability and promoted migration, and decreased the apoptotic rate of hypopharyngeal carcinoma cells, compared with the negative control-transfected cells (Fig. 2B-H).

Overexpression of miRNA-98 increases the apoptotic rate and inhibits the cell growth and migration of hypopharyngeal carcinoma cells. Next, we studied the function of miRNA-98 
A

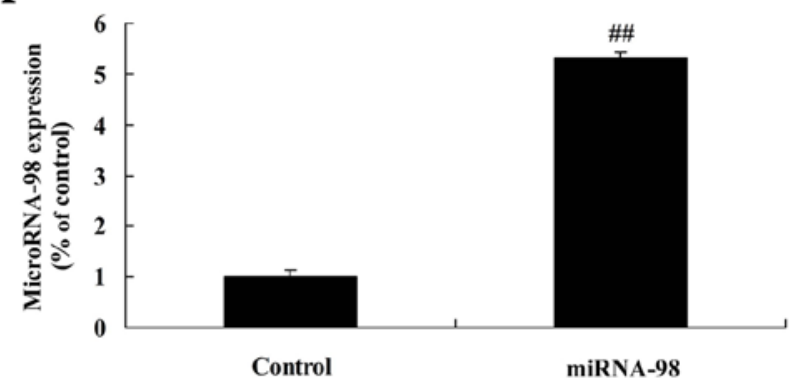

C

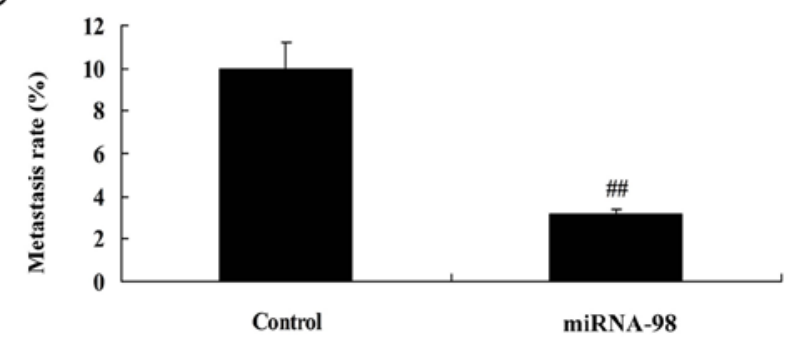

E

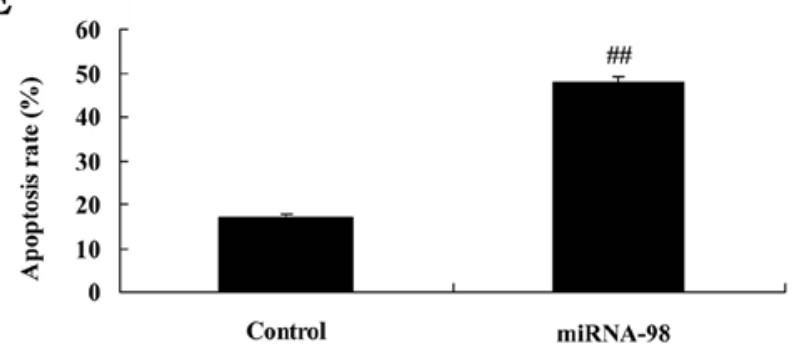

G

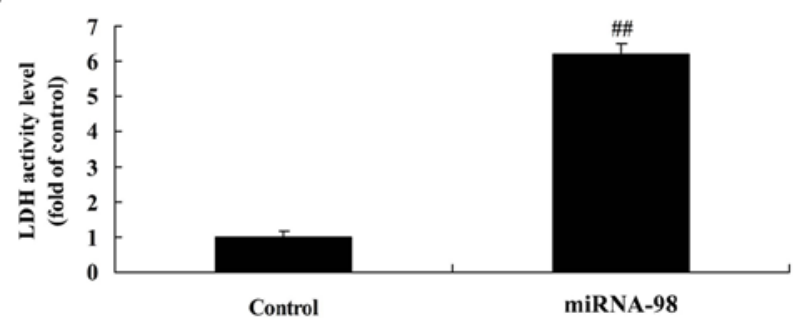

B

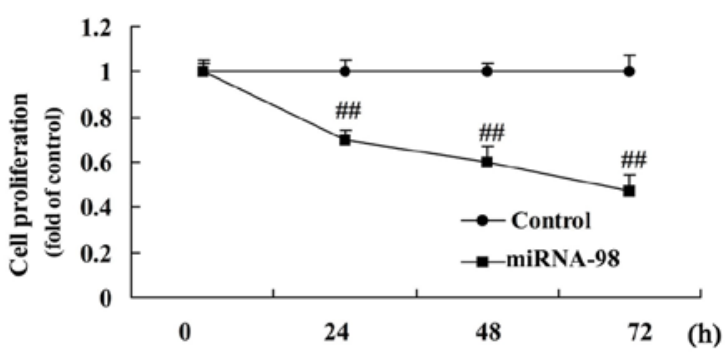

D
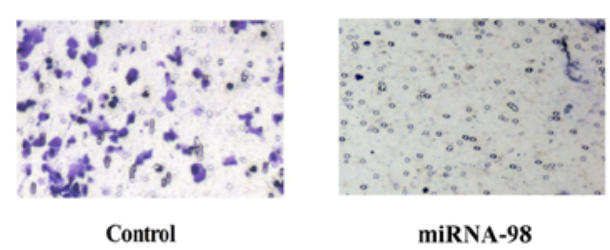

F
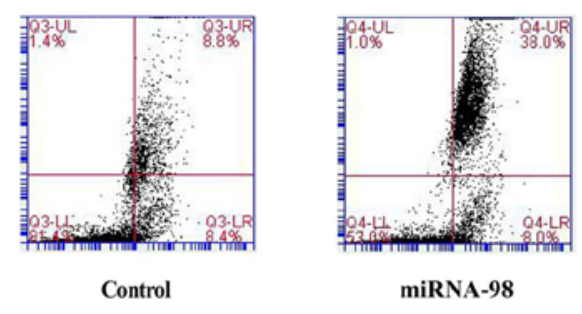

miRNA-98

$\mathrm{H}$

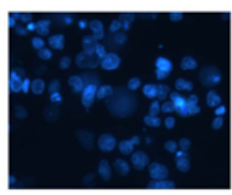

Control

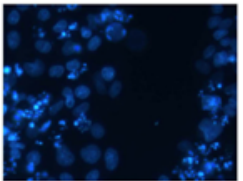

miRNA-98

Figure 3. Overexpression of microRNA-98 increased the apoptosis rate and inhibits cell growth and migration of hypopharyngeal carcinoma cells. (A) MicroRNA-98 expression, (B) cell proliferation, (C and D) cell migratory, (E and F) the apoptosis rate, (G) LDH activity and (H) DAPI assay. Control, negative control group; miRNA-98, microRNA-98 overexpression group. ${ }^{\# \#} \mathrm{P}<0.01$ compared with the negative control group.

in hypopharyngeal carcinoma. FaDu cells were transiently transfected with micRNA-98 mimics, and miRNA-98 expression was substantially increased in the FaDu cells, compared with that noted in the negative control-transfected cells (Fig. 3A). Overexpression of miRNA-98 enhanced the apoptotic rate and inhibited cell growth and migration of hypopharyngeal carcinoma cells, compared with the negative control-transfected cells (Fig. 3B-H).

miRNA-98 regulates the PTEN/AKT pathway by MTDH. To measure the mechanism of miRNA-98 in regards to the apoptosis of hypopharyngeal carcinoma, we analyzed the changes in the pathways in hypopharyngeal carcinoma cell lines with overexpression of miRNA-98. Heat map showed that MTDH expression was suppressed, while PTEN expression was increased in the hypopharyngeal carcinoma cell line following overexpression of miRNA-98 (Fig. 4A). Then, the reporter assay showed that MTDH is a target of miRNA-98, and overexpression of miRNA-98 decreased the activity of reporter assay levels, compared with the negative group (Fig. 4B and C). Moreover, immunofluorescence (IF) showed that overexpression of miRNA-98 suppressed the protein expression of MTDH in hypopharyngeal carcinoma cells, compared with that noted in the negative control-transfected cells (Fig. 4D). Overexpression of miRNA-98 suppressed the protein expression of MTDH and p-Akt while it induced that of PTEN and Bax in hypopharyngeal carcinoma cells, compared with the negative control-transfected cells (Fig. 4E-I). Additionally, overexpression of miRNA-98 promoted caspase-3/9 activity levels in the hypopharyngeal 

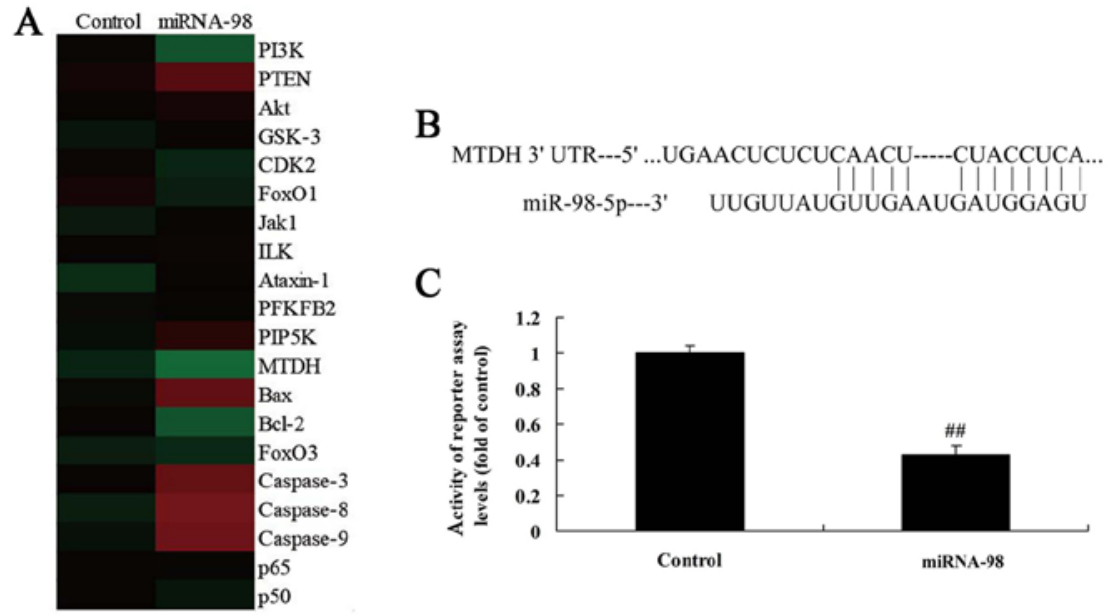

C

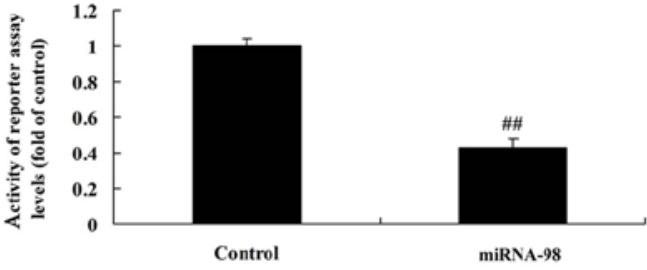

$\mathrm{D}$

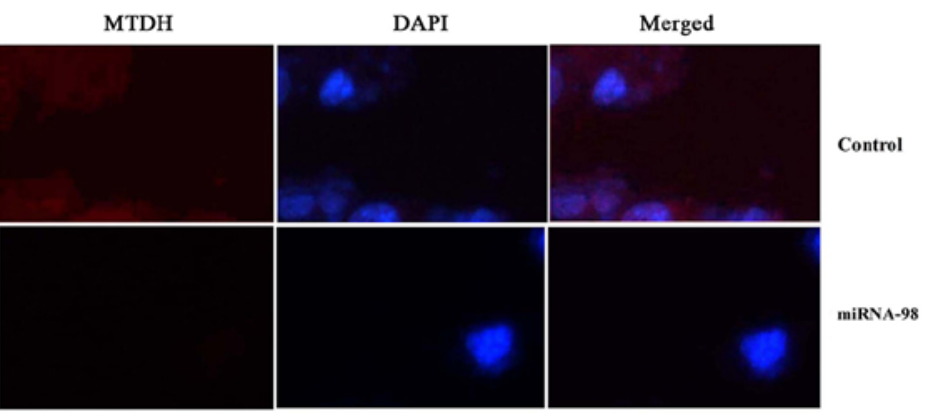

\section{$\mathrm{E}$}
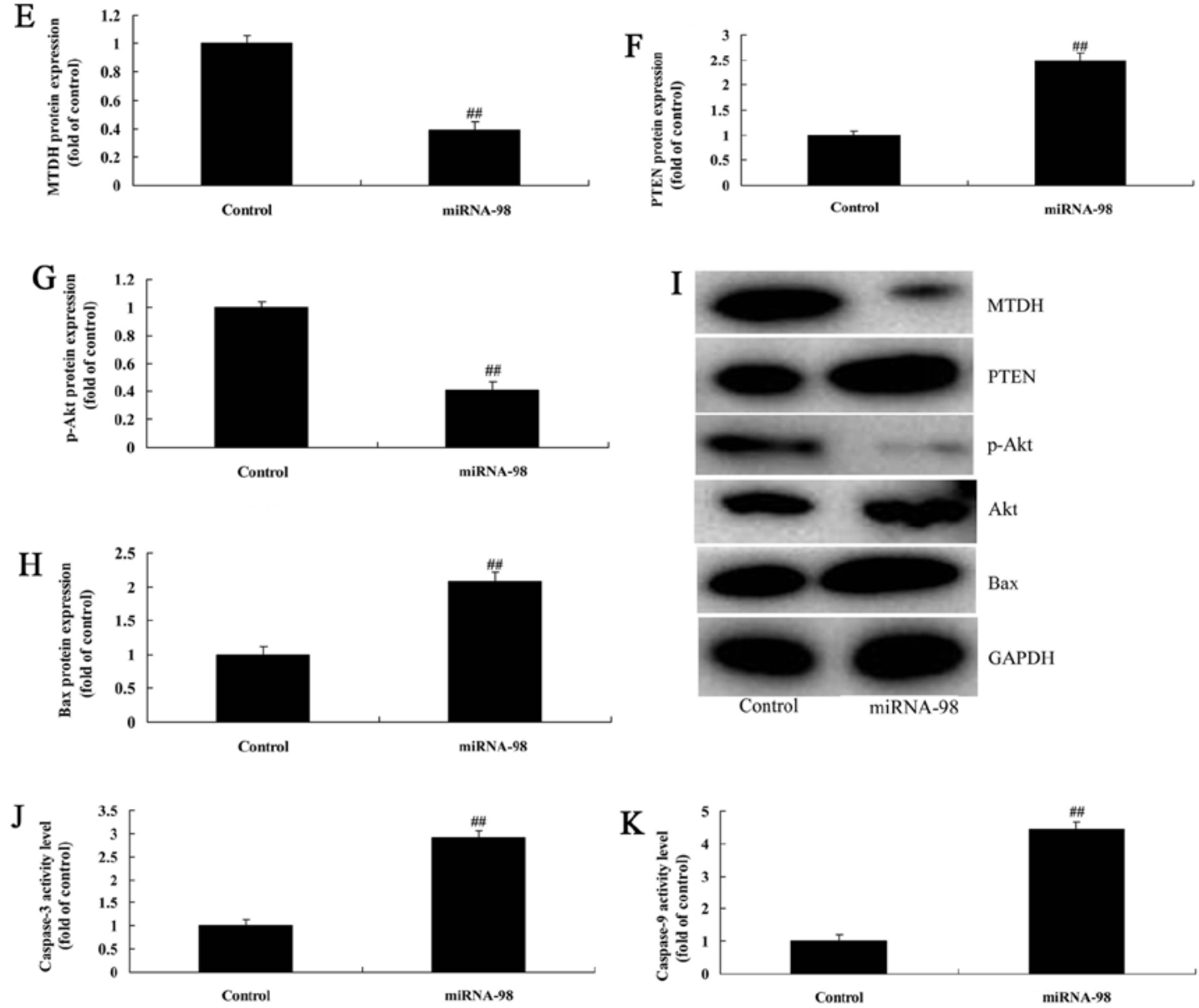

Figure 4. Overexpression of miRNA-98 regulates the PTEN/AKT pathway by MTDH. (A) Heat map. (B) MTDH is a target of microRNA-98. (C) Activity of reporter assay levels. (D) Immunofluorescence for MTDH protein expression. (E) MTDH, (G) p-Akt, (F) PTEN and (H) Bax protein expression as determined by statistical analysis. (I) Western blot assays for MTDH, p-Akt, PTEN and Bax protein expression. (J and K) Caspase-3 and caspase-9 activity levels. Control, negative control group; miRNA-98, of microRNA-98 overexpression group. ${ }^{\# \#} \mathrm{P}<0.01$ compared with the negative control group. 
A

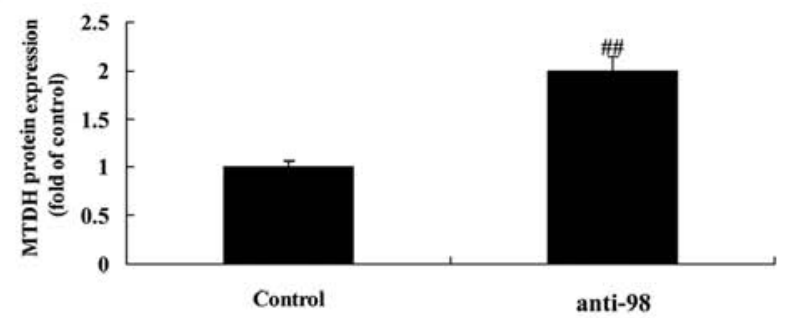

C

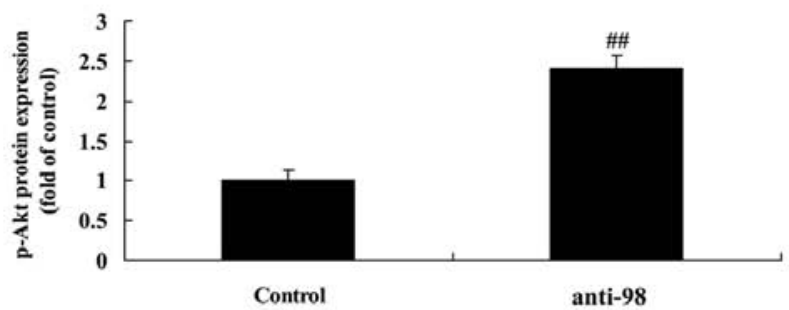

D

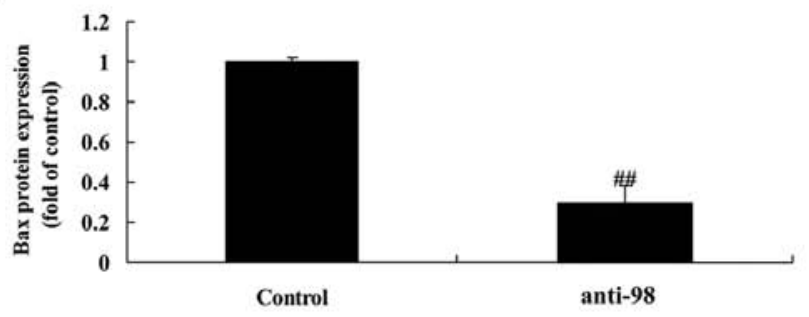

F

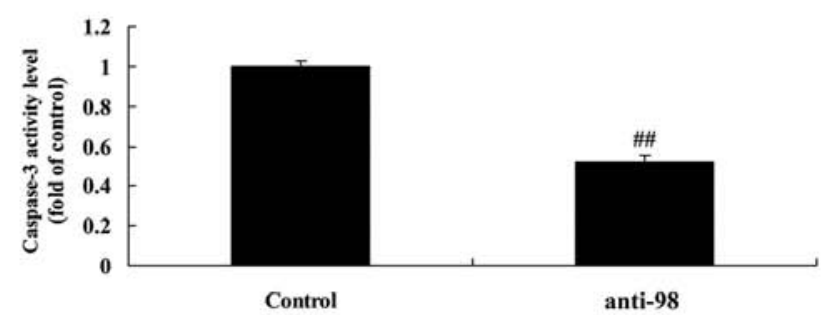

B

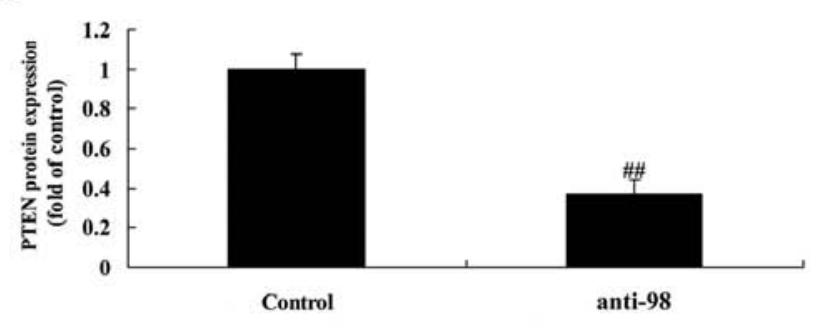

E

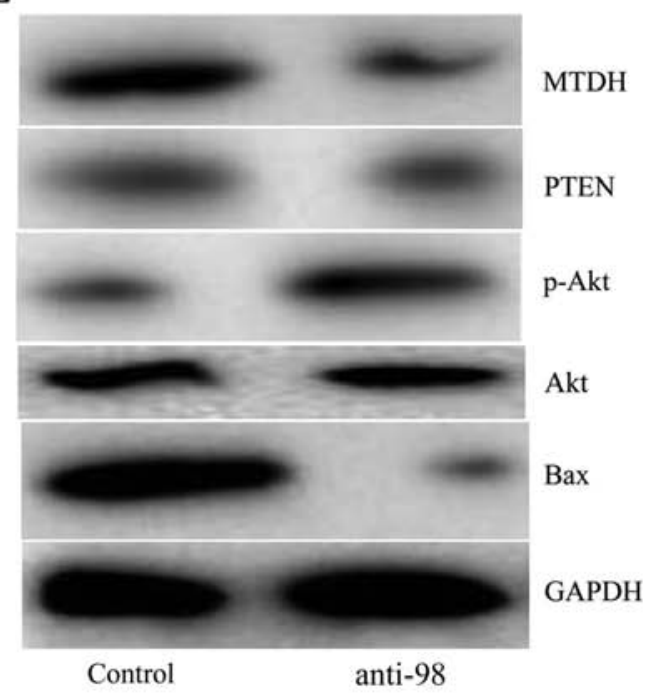

G

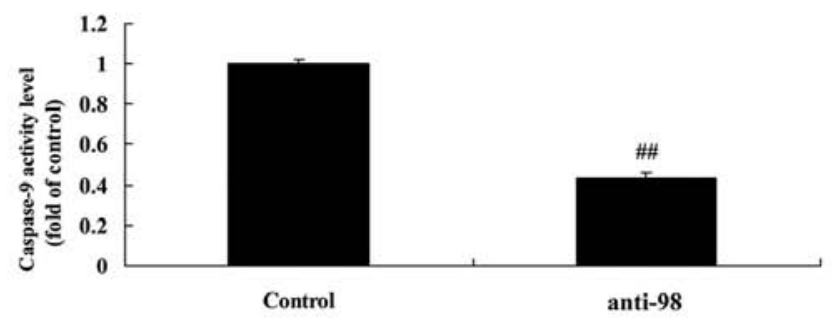

Figure 5. Downregulation of miRNA-98 regulates the PTEN/AKT pathway by MTDH. (A-D) MTDH, p-Akt, PTEN and Bax protein expression as determined by statistical analysis. (E) Western blot assays for MTDH, p-Akt, PTEN and Bax protein expression. (F and G) Caspase-3 and caspase-9 activity levels. Control, negative control group; Anti-98, microRNA-98 downregulated expression group. ${ }^{\# \#} \mathrm{P}<0.01$ compared with the negative control group.

carcinoma cells, compared with the negative control-transfected cells (Fig. 4J and K). However, downregulation of miRNA-98 induced the protein expression of MTDH and p-Akt protein (Fig. 5A, C and E), while inhibited that of PTEN and Bax (Fig. 5B, D and E), and reduced caspase-3/9 activity levels (Fig. 5F and G) in hypopharyngeal carcinoma cells, compared with the negative control-transfected cells. These results showed that miRNA-98 induced apoptosis of hypopharyngeal carcinoma by regulating the PTEN/AKT/caspase-3/9 pathway via MTDH.

MTDH attenuates the anticancer function of miRNA-98 in hypopharyngeal carcinoma via the PTEN/AKT pathway. We further studied the role of MTDH in the anticancer function of miRNA-98 in hypopharyngeal carcinoma via the PTEN/AKT pathway. As shown in Fig. 6, the MTDH plasmid induced the protein expression of MTDH and p-Akt, while suppressing that of PTEN and Bax, inhibited caspase-3/9 activity levels in hypopharyngeal carcinoma by miRNA-98, compared with the miRNA-98 group without MTDH plasmid treatment. Then, the activation of MTDH attenuated the anticancer effects of miRNA-98 on the inhibition of cell viability and migration, and the promotion of the apoptotic rate and $\mathrm{LDH}$ activity levels in hypopharyngeal carcinoma by miRNA-98, compared with the miRNA-98 group without the activation of MTDH (Fig. 7). These results indicate that MTDH attenuated the anticancer effects of miRNA-98 in hypopharyngeal carcinoma via the PTEN/AKT pathway.

Inhibition of PTEN attenuates the anticancer effects of miRNA-98 in hypopharyngeal carcinoma via the PTEN/AKT pathway. Finally, we investigated the function of PTEN in the anticancer effects of miRNA-98 in hypopharyngeal carcinoma via the PTEN/AKT pathway. The administration 
A
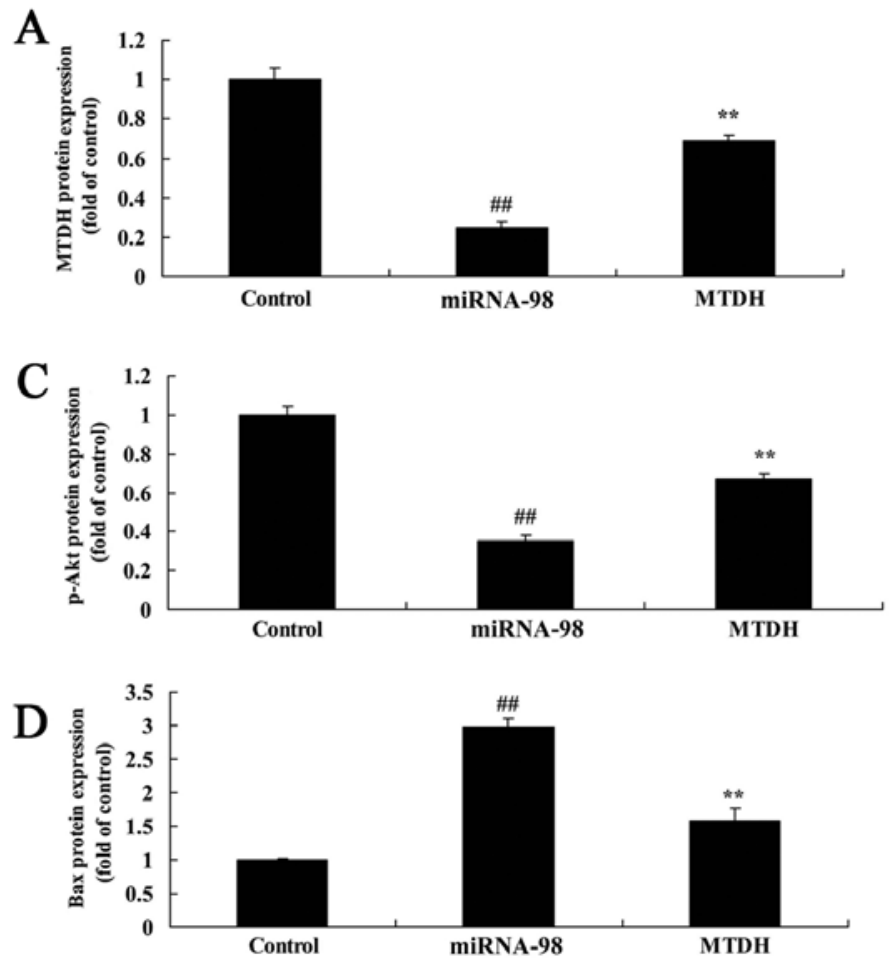

F

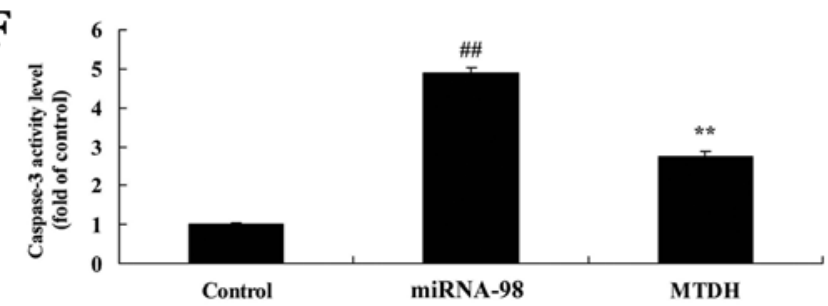

B

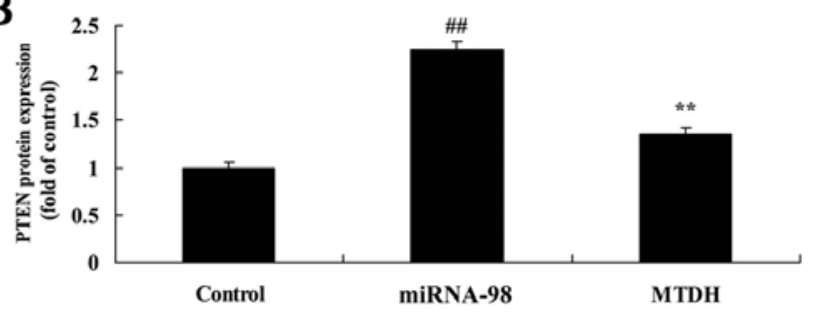

E

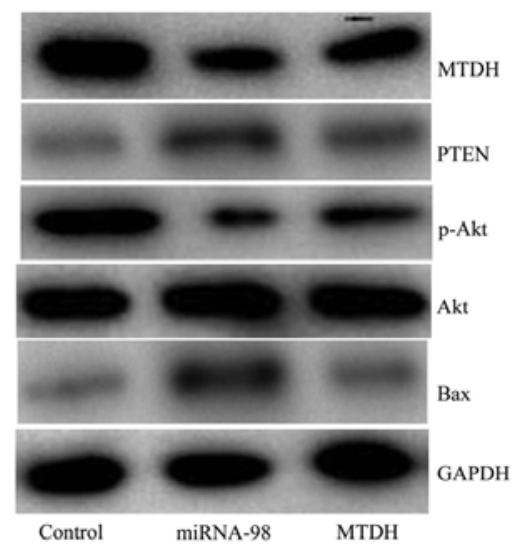

G

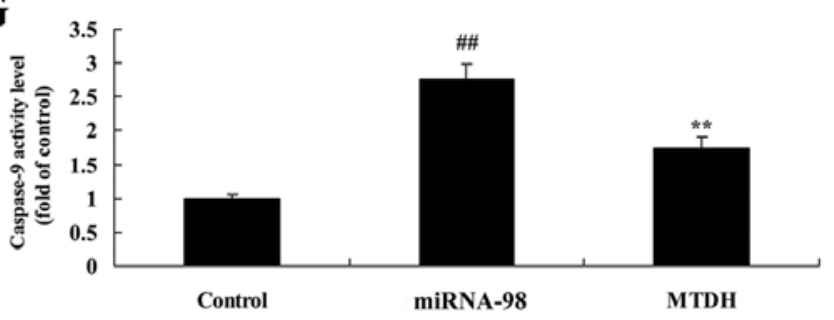

Figure 6. MTDH decreases the anticancer function of miRNA-98 in hypopharyngeal carcinoma via the PTEN/AKT pathway. (A-D) MTDH, p-Akt, PTEN and Bax protein expression as determined by statistical analysis. (E) Western blot assays of MTDH, p-Akt, PTEN and Bax protein expression. (F and G) Caspase-3 and caspase-9 activity levels. Control, negative control group; miRNA-98, microRNA-98 overexpression group; MTDH, microRNA-98 overexpression and MTDH group. ${ }^{\# \#} \mathrm{P}<0.01$ compared with the negative control group, ${ }^{* *} \mathrm{P}<0.01$ compared with the microRNA-98 overexpression group.

of PTEN inhibitor (20 nM, VO-Ohpic trihydrate) suppressed the protein expression of PTEN and Bax, inhibited caspase-3/9 activity levels, and promoted p-AKT protein expression in hypopharyngeal carcinoma by miRNA-98, compared with the miRNA-98 group without PTEN inhibitor treatment (Fig. 8). Then, the inhibition of PTEN attenuated the anticancer effects of miRNA-98 on the inhibition of cell viability and migration, and the promotion of the apoptotic rate and LDH activity levels in hypopharyngeal carcinoma by miRNA-98, compared with the miRNA-98 group without PTEN inhibition (Fig. 9). These results indicated that miRNA-98 inhibited the proliferation and induced apoptosis in hypopharyngeal carcinoma cells via the PTEN/AKT pathway by MTDH.

\section{Discussion}

Early diagnosis of laryngeal carcinoma and improvement of treatment efficacy for laryngeal carcinoma is vital. In addition, the further understanding of the mechanisms underlying the genesis and development of laryngeal carcinoma are needed (13). This will contribute to the identification of novel treatment methods or early diagnosis and treatment (13). Epidemiological investigation has confirmed that smoking, alcohol abuse, air pollution and occupational factors are the causes of laryngeal carcinoma (14). Molecular biological research has been intensively carried out. Basic research on laryngeal carcinoma-related miRNAs has displayed their valuable application prospect as laryngeal carcinoma treatment targets (15). On the one hand, an in vitro study verify that some miRNAs can suppress laryngeal carcinoma cell proliferation. For instance, increasing the miR-34c expression level can effectively suppress laryngeal carcinoma cell proliferation (16). This reveals that miR-34c a promising potential target for treating laryngeal carcinoma. On the other hand, an in vivo study confirmed that injection of miR-21 antisense nucleotide chain lentiviral vector in xenografts of squamous carcinoma of the larynx can effectively suppress laryngeal carcinoma cell growth (16). The present study showed that OS and DFS of patients with hypopharyngeal carcinoma in a high microRNA-98 expression were prolonged when compared with that of patients with hypopharyngeal carcinoma in a 
A

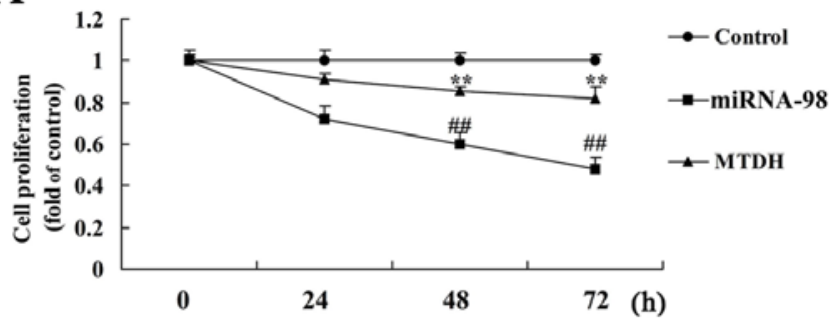

B

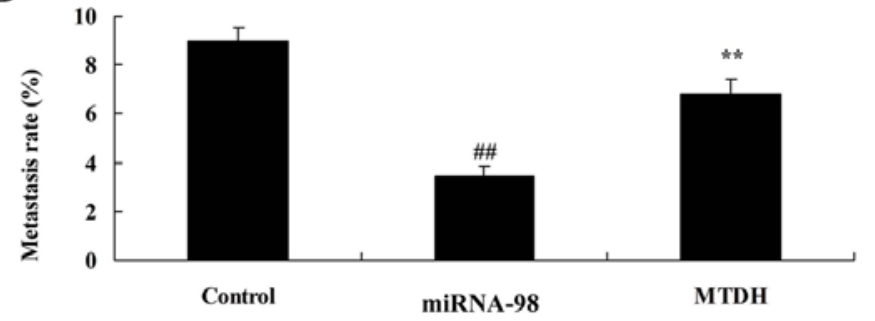

C

D

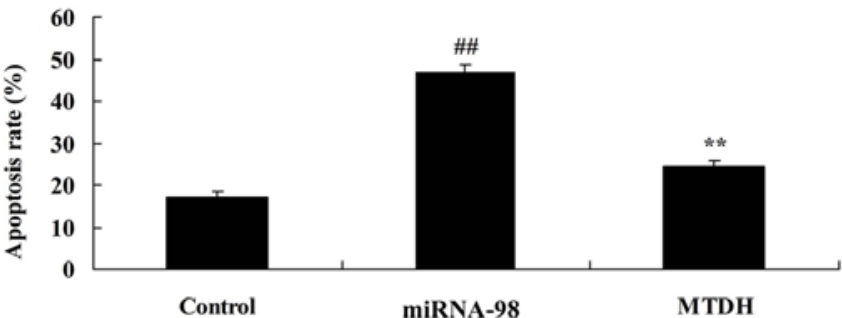

E
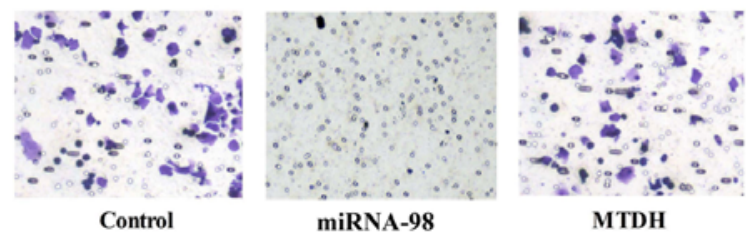

F

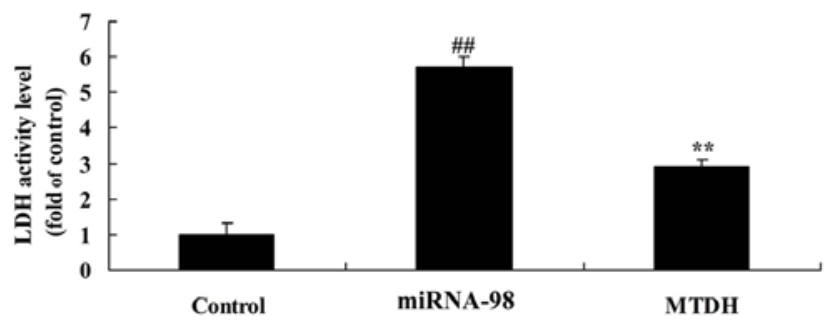

G

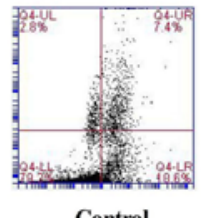

Control

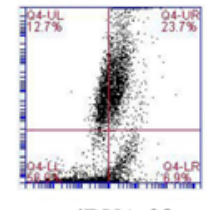

miRNA-98

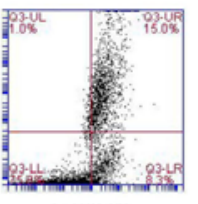

MTDH

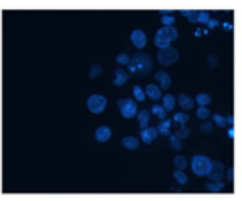

Control

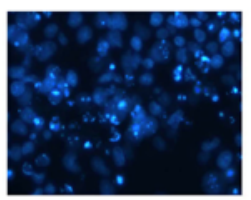

miRNA-98

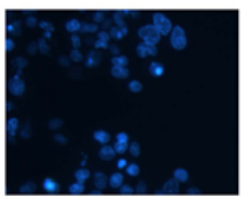

MTDH

Figure 7. MTDH decreases the anticancer function of miRNA-98 in hypopharyngeal carcinoma cell growth. (A) Cell growth, (B and C) cell migration, (D and E) apoptosis rate, (F) LDH activity and (G) DAPI assay. Control, negative control group; miRNA-98, microRNA-98 overexpression group; MTDH, microRNA-98 overexpression and MTDH group. ${ }^{\# \#} \mathrm{P}<0.01$ compared with the negative control group, ${ }^{* *} \mathrm{P}<0.01$ compared with the microRNA-98 overexpression group.

low microRNA-98 expression group. Downregulation of microRNA-98 promoted cell growth and migratory, and decreased the apoptosis rate of hypopharyngeal carcinoma cells. Wang et al showed that let-7/miR-98 regulate Fas and Fas-mediated apoptosis (17).

It was reported that MTDH is expressed in almost all malignant tumor cells. MTDH overexpression can enhance the proliferation, differentiation, metastasis and invasion capacity of endometrial cancer, prostate cancer, breast cancer and human glioma cells (18). In addition, MTDH plays a key role in upregulating tumor genesis mediated by the Ha-as oncogene (19). Blocking MTDH expression can upregulate (FOXO)3A activity to induce apoptosis (20). MTDH silencing was found to notably restrain human neuroblastoma cell proliferation and induce apoptosis. As a result, cells were arrested at the G0/G1 stage of the cell cycle (20). MTDH silencing was found to enhance cancer cell sensitivity to chemotherapeutics such as cisplatin and doxorubicin. Thus, it can effectively inhibit tumor cell proliferation and promote cell apoptosis (20). In the present study, it was found that MTDH decreased the anticancer function of microRNA-98 in hypopharyngeal carcinoma via the PTEN/AKT pathway. Wang et al reported that the upregulation of miR-98 inhibited apoptosis in cartilage cells in osteoarthritis (21).

The PI3K/Akt pathway is a major branch of the receptor tyrosine kinase pathway. It is an important growth factor pathway in vivo (7). It can activate the anti-cell apoptosis mechanism, glucose metabolism and protein synthesis and thus enhance cell proliferation and growth (22). The PDK/Akt signal transduction pathway is abnormally activated in multiple malignant tumors (7). The following two mechanisms have been extensively studied. One is tyrosine kinase-mediated PI3K activation. For instance, the phosphorylated tyrosine kinase binds with subunit p85, or the mutant Ras directly binds with EBK to activate PI3K. The other one is somatic gene mutation (22). 
A

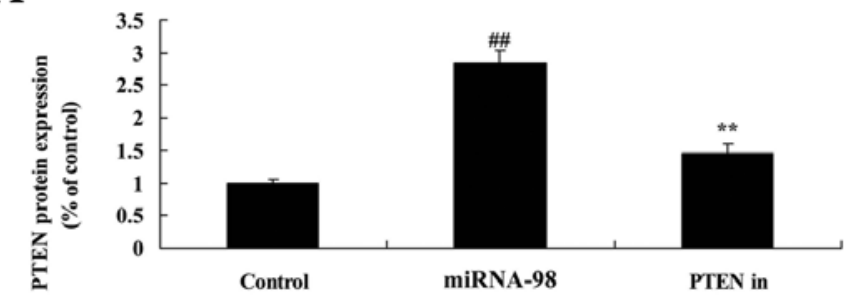

C

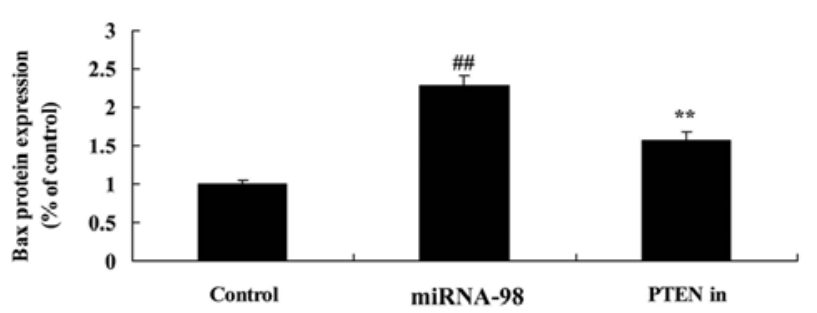

E

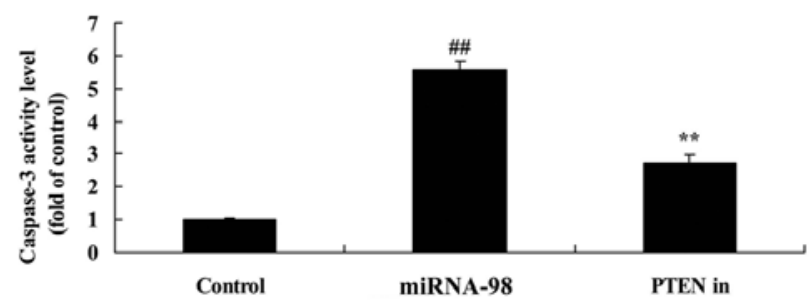

F

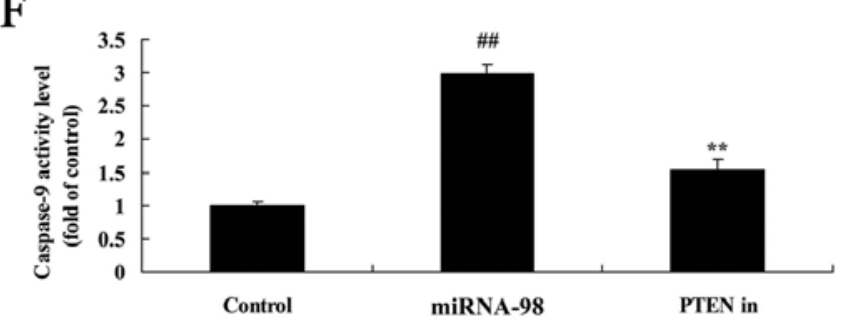

B

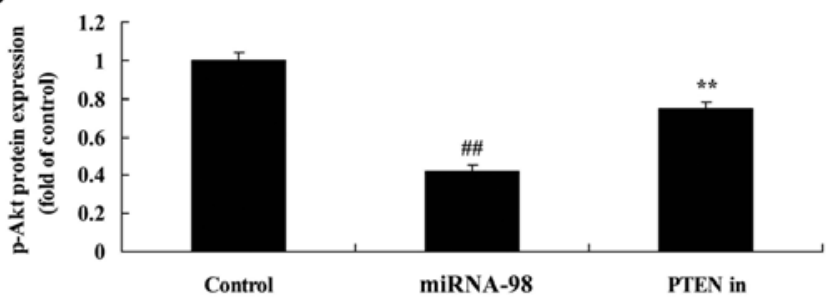

$\mathrm{D}$

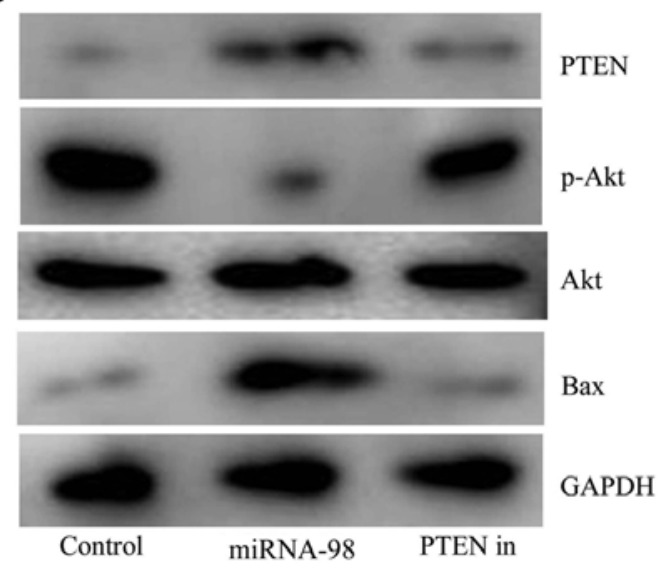

Figure 8. Inhibition of PTEN reduces the anticancer effects of miRNA-98 in hypopharyngeal carcinoma via the PTEN/AKT pathway. (A-C) p-Akt, PTEN and Bax protein expression as determined by statistical analysis. (D) Western blot assays of p-Akt, PTEN and Bax protein expression. (E and F) Caspase-3 and caspase-9 activity levels. Control, negative control group; miRNA-98, microRNA-98 overexpression group; PTEN in, microRNA-98 overexpression and PTEN inhibitor group. ${ }^{\# \#} \mathrm{P}<0.01$ compared with the negative control group, ${ }^{* *} \mathrm{P}<0.01$ compared with the microRNA-98 overexpression group.

For instance, PTEN tumor-suppressor gene mutation makes it impossible to block the PI3K pathway. It was recently discovered that the PIK3CA gene mutation occurs in over $30 \%$ of epithelial tumors (23). The above-mentioned tyrosine kinase-mediated abnormal PI3K activation and somatic gene mutation can induce abnormal cell proliferation. In this way, it can induce the genesis and development of malignant tumors such as ovarian, breast, pancreatic, lung and colon cancer (23). The present study showed that overexpression of miRNA-98 suppressed MTDH and p-Akt protein expression and induced PTEN protein expression in hypopharyngeal carcinoma cells. Chen et al showed that miRNA-98 rescues proliferation and alleviates ox-LDL-induced apoptosis in human umbilical vein ECs (HUVECs) by targeting LOX-1 (24).

PTEN is the first tumor-suppressor gene discovered to date to possess phosphatase activity. The major tumor-suppressing effect of PTEN is to promote apoptosis (25). PTEN gene mutations render loss of all gene functions (25). Thus, cell growth is out of control, and apoptosis cannot be carried out in time. Low PTEN expression in laryngeal carcinoma suggests reduced apoptosis, enhanced growth and improved invasion. The PTEN gene can suppress tumor transcription. Moreover, it is related to tumor pathological differentiation and prognosis (26). Notably, in the present study, the promotion of MTDH or the inhibition of PTEN reduced the anticancer effects of miRNA-98 in hypopharyngeal carcinoma via the PTEN/AKT pathway. This study only analyzed one cell line, and it is a limitation of this study. We will use more cell models or a mouse model in further studies.

In conclusion, the function of miRNA-98 inhibits hypopharyngeal carcinoma cell proliferation and induces apoptosis via the PTEN/AKT pathway by MTDH. Moreover, the miRNA-98/ PTEN/AKT signaling pathway may serve a key role in the anticancer effects of hypopharyngeal carcinoma. 
A

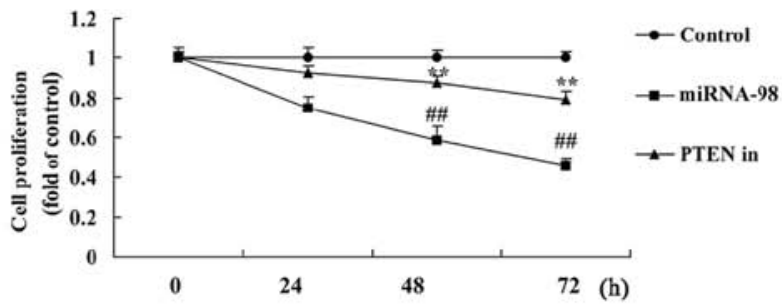

B

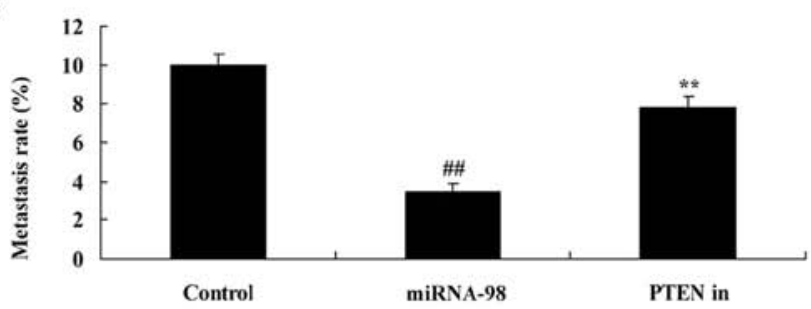

D

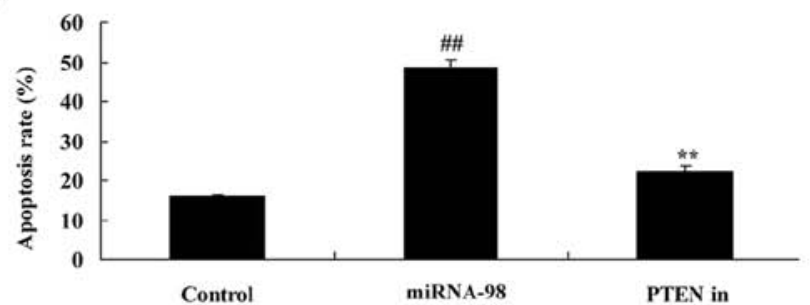

F

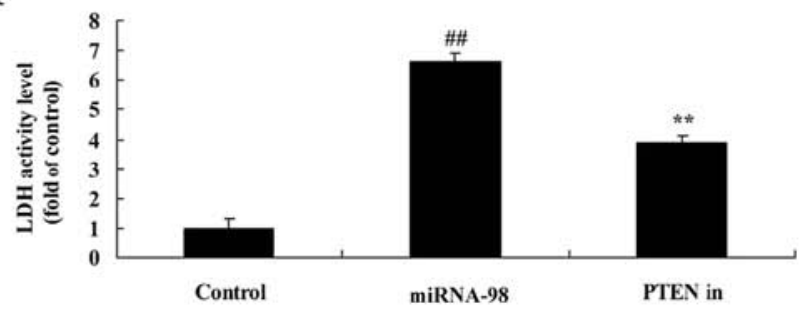

C

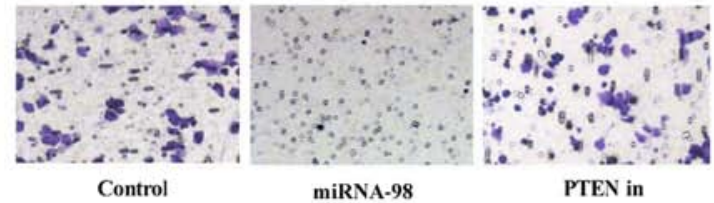

$\mathrm{E}$
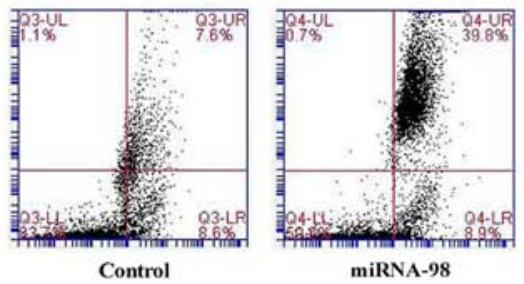

miRNA-98

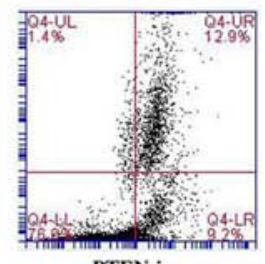

PTEN in

G
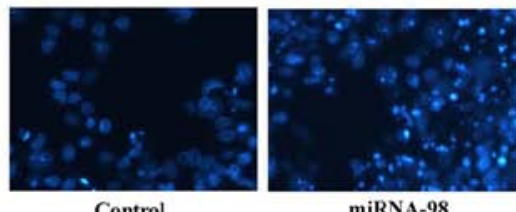

miRNA-98

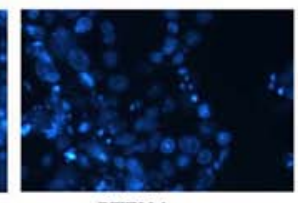

PTEN in

Figure 9. Inhibition of PTEN reduces the anticancer effects of miRNA-98 in hypopharyngeal carcinoma. (A) Cell growth, (B and C) cell migration,(D and E) apoptosis rate, (F) LDH activity and (G) DAPI assay. Control, negative control group; miRNA-98, microRNA-98 overexpression group; PTEN in, microRNA-98

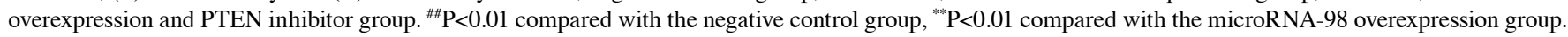

\section{Acknowledgements}

Not applicable.

\section{Funding}

The present study was supported by the Natural Science Foundation (grant no. H2017033) and the Health and Family Planning Commission (no. 2018019) of Heilongjiang Province of China.

\section{Availability of data and materials}

The datasets used during the present study are available from the corresponding author upon reasonable request.

\section{Authors' contributions}

JL designed the experiments; QW, LT, JZ, XZ and TY performed the experiments and collected the data; $\mathrm{QW}$ and
JL analyzed the data; JL wrote the manuscript. All authors read and approved the manuscript and agree to be accountable for all aspects of the research in ensuring that the accuracy or integrity of any part of the work are appropriately investigated and resolved.

\section{Ethics approval and consent to participate}

The study protocol was approved by the Human Research Ethics Committee of The First Affiliated Hospital of Harbin Medical University, and all patients provided written informed consent prior to participation in the present study.

\section{Patient consent for publication}

Not applicable.

\section{Competing interests}

The authors state that they have no competing interests. 


\section{References}

1. Nakashima T, Yasumatsu R, Asai K, Uryu H, Kogo R and Nakagawa T: Single-cycle induction chemotherapy for resectable advanced hypopharyngeal cancer. Int J Clin Oncol 22: 442-447, 2017.

2. Bonner J, Giralt J, Harari P, Spencer S, Schulten J, Hossain A, Chang SC, Chin S and Baselga J: Cetuximab and radiotherapy in laryngeal preservation for cancers of the larynx and hypopharynx: A secondary analysis of a randomized clinical trial. JAMA Otolaryngol Head Neck Surg 142: 842-849, 2016.

3. Damascelli B, Patelli G, Ticha V, Di Tolla G, Frigerio LF, Garbagnati F, Lanocita R, Marchianò A, Spreafico C, Mattavelli F, et al: Feasibility and efficacy of percutaneous transcatheter intraarterial chemotherapy with paclitaxel in albumin nanoparticles for advanced squamous-cell carcinoma of the oral cavity, oropharynx, and hypopharynx. J Vasc Interv Radiol 18: 1395-1403, 2007.

4. Yoo GH, Moon J, Leblanc M, Lonardo F, Urba S, Kim H Hanna E, Tsue T, Valentino J, Ensley J and Wolf G: A phase 2 trial of surgery with perioperative INGN 201 (Ad5CMV-p53) gene therapy followed by chemoradiotherapy for advanced, resectable squamous cell carcinoma of the oral cavity, oropharynx, hypopharynx, and larynx: Report of the Southwest Oncology Group. Arch Otolaryngol Head Neck Surg 135: 869-874, 2009.

5. Hess AK, Müer A, Mairinger FD, Weichert W, Stenzinger A, Hummel M, Budach V and Tinhofer I: MiR-200b and miR-155 as predictive biomarkers for the efficacy of chemoradiation in locally advanced head and neck squamous cell carcinoma. Eur J Cancer 77: 3-12, 2017.

6. Wang L, Mou Y, Meng D, Sun Y, Chen X, Yang X, Jia C, Song X and Li X: MicroRNA-203 inhibits tumour growth and metastasis through PDPN. Clin Otolaryngol 42: 620-628, 2017.

7. Yang N, Hui L, Wang Y, Yang $H$ and Jiang X: SOX2 promotes the migration and invasion of laryngeal cancer cells by induction of MMP-2 via the PI3K/Akt/mTOR pathway. Oncol Rep 31: 2651-2659, 2014.

8. Si F, Sun J and Wang C: MicroRNA-138 suppresses cell proliferation in laryngeal squamous cell carcinoma via inhibiting EZH2 and PI3K/AKT signaling. Exp Ther Med 14: 1967-1974, 2017.

9. Wang B, Qin H, Wang Y, Chen W, Luo J, Zhu X, Wen W and Lei W: Effect of DJ-1 overexpression on the proliferation, apoptosis, invasion and migration of laryngeal squamous cell carcinoma SNU-46 cells through PI3K/AKT/mTOR. Oncol Rep 32: 1108-1116, 2014.

10. Zhang Y, Wang Y and Wang J: MicroRNA-584 inhibits cell proliferation and invasion in non-small cell lung cancer by directly targeting MTDH. Exp Ther Med 15: 2203-2211, 2018.

11. Liu J, Lei DP, Jin T, Zhao XN, Li G and Pan XL: Altered expression of miR-21 and PTEN in human laryngeal and hypopharyngeal squamous cell carcinomas. Asian Pac J Cancer Prev 12: 2653-2657, 2011.

12. Livak KJ and Schmittgen TD: Analysis of relative gene expression data using real-time quantitative PCR and the 2(-Delta Delta C(T)) method. Methods 25: 402-408, 2001.

13. Elliott EA, Wright JR, Swann RS, Nguyen-Tân F, Takita C, Bucci MK, Garden AS, Kim H, Hug EB, Ryu J, et al: Phase III Trial of an emulsion containing trolamine for the prevention of radiation dermatitis in patients with advanced squamous cell carcinoma of the head and neck: Results of radiation therapy oncology group trial 99-13. J Clin Oncol 24: 2092-2097, 2006.
14. Durmus K, Kucur C, Uysal IO, Dziegielewski PT and Ozer E: Feasibility and clinical outcomes of transoral robotic surgery and transoral robot-assisted carbon dioxide laser for hypopharyngeal carcinoma. J Craniofac Surg 26: 235-237, 2015.

15. Orosz E, Gombos K, Riedling T, Afiakurue P, Kiss I, Pytel J, Gerlinger I and Szanyi I: Comparative miRNA expression profile analysis of squamous cell carcinoma and peritumoral mucosa from the meso- and hypopharynx. Cancer Genomics Proteomics 14: 285-292, 2017.

16. Re M, Magliulo G, Gioacchini FM, Bajraktari A, Bertini A, Çeka A, Rubini C, Ferrante L, Procopio AD and Olivieri F: Expression levels and clinical significance of miR-21-5p, miR-let-7a, and miR-34c-5p in laryngeal squamous cell carcinoma. Biomed Res Int 2017: 3921258, 2017.

17. Wang S, Tang Y, Cui H, Zhao X, Luo X, Pan W, Huang X and Shen N: Let-7/miR-98 regulate Fas and Fas-mediated apoptosis. Genes Immun 12: 149-154, 2011.

18. Li J, Li C, Li H, Zhang T, Hao X, Chang J and Xu Y: MicroRNA-30a-5p suppresses tumor cell proliferation of human renal cancer via the MTDH/PTEN/AKT pathway. Int J Mol Med 41: 1021-1029, 2018.

19. Mizrak Kaya D, Dong X, Nogueras-González GM, Xu Y, Estrella JS, Harada K, Lopez A, Amlashi FG, Hofstetter WL, Maru DM, et al: Post-trimodality expression levels of metadherin (MTDH) as a prognostic biomarker for esophageal adenocarcinoma patients. Med Oncol 34: 135, 2017.

20. Qiao W, Cao N and Yang L: MicroRNA-154 inhibits the growth and metastasis of gastric cancer cells by directly targeting MTDH. Oncol Lett 14: 3268-3274, 2017.

21. Wang GL, Wu YB, Liu JT and Li CY: Upregulation of miR-98 inhibits apoptosis in cartilage cells in osteoarthritis. Genet Test Mol Biomarkers 20: 645-653, 2016.

22. Luo XM, Xu B, Zhou ML, Bao YY, Zhou SH, Fan J and Lu ZJ: Co-Inhibition of GLUT-1 expression and the PI3K/Akt signaling pathway to enhance the radiosensitivity of laryngeal carcinoma xenografts in vivo. PLoS One 10: e0143306, 2015.

23. Zhang XJ and Jia SS: Fisetin inhibits laryngeal carcinoma through regulation of $\mathrm{AKT} / \mathrm{NF}-\kappa \mathrm{B} / \mathrm{mTOR}$ and $\mathrm{ERK} 1 / 2$ signaling pathways. Biomed Pharmacother 83: 1164-1174, 2016.

24. Chen Z, Wang M, He Q, Li Z, Zhao Y, Wang W, Ma J, Li Y and Chang G: MicroRNA-98 rescues proliferation and alleviates ox-LDL-induced apoptosis in HUVECs by targeting LOX-1. Exp Ther Med 13: 1702-1710, 2017.

25. Zhu XL, Wang ZF, Lei WB, Zhuang HW, Hou WJ, Wen YH and Wen WP: Tumorigenesis role and clinical significance of DJ-1, a negative regulator of PTEN, in supraglottic squamous cell carcinoma. J Exp Clin Cancer Res 31: 94, 2012.

26. Wang S, Guo D and Li C: Downregulation of miRNA-26b inhibits cancer proliferation of laryngeal carcinoma through autophagy by targeting ULK2 and inactivation of the PTEN/AKT pathway. Oncol Rep 38: 1679-1687, 2017.

This work is licensed under a Creative Commons Attribution-NonCommercial-NoDerivatives 4.0 International (CC BY-NC-ND 4.0) License. 